\title{
How Can a Hydrophobic MOF be Water-Unstable? Insight into the Hydration Mechanism of IRMOFs
}

\author{
Marta De Toni, ${ }^{a, b}$ Romain Jonchiere, ${ }^{a, \dagger}$ Dr. Pluton Pullumbi, ${ }^{b}$ \\ Dr. F.-X. Coudert, ${ }^{a} *$ Prof. Dr. Alain H. Fuchs ${ }^{a}$ \\ ${ }^{a}$ Chimie ParisTech \& CNRS, 11 rue Pierre et Marie Curie, 75005 Paris, France. \\ ${ }^{\mathrm{b}}$ Air Liquide, Centre de Recherche Claude Delorme, 78354 Jouy-en-Josas, France. \\ $\dagger$ Presently at: IMPMC, CNRS-IPGP-UPMC, 75005 Paris, France. \\ Author email address: fx.coudert@chimie-paristech.fr
}

\begin{abstract}
.
We report an ab initio Molecular Dynamics study of the hydration process in a model IRMOF material. At low water content (one molecule per unit cell), water physisorption is observed on the zinc cation but the free $\rightleftarrows$ bound equilibrium strongly favors the free state. This is consistent with the hydrophobic nature of the host matrix and its type $\mathrm{V}$ isotherm observed in a classical Monte Carlo simulation. At higher loading, a water cluster can be formed at the $\mathrm{Zn}_{4} \mathrm{O}$ site and this is shown to stabilize the water bound state. This structure very rapidly transforms into a linkerdisplaced state, where water has fully displaced one arm of a linker and which corresponds to the loss of the material's fully-ordered structure. Thus an overall hydrophobic MOF material can also become water unstable, a feature that was not fully understood until now.
\end{abstract}

Keywords: molecular dynamics, ab initio calculation, water, reaction mechanisms, metal-organic frameworks

\section{Introduction.}

Nanoporous metal-organic frameworks (MOFs) display a large range of crystal structures and host-guest properties, due to a combination of tunable porosity, by choice of metal centers and linker length, and functionalisation of the internal surface of the material. Among the proposed applications of MOFs, adsorptive storage and separation of strategic gases $\left(\mathrm{H}_{2}, \mathrm{CO}_{2}, \mathrm{CH}_{4}\right)$ are of particular importance and have gained a lot of attention in recent years. ${ }^{1,2}$ Although a very large number of materials with varying structures and chemistry have been synthesized, and the adsorption of a handful of gases is routinely performed along with structure characterization, there 
is still relatively little data available on water adsorption in MOFs. ${ }^{3}$ It is of great importance to understand the interaction of water with these materials, so as to better predict how they behave in real-life conditions, how they will age and keep (or loose) their surface area and adsorption properties over time. While some MOFs are stable in the presence of water, even when left in liquid water for long periods of time, others are unstable in the presence of water vapor or moist air. Such water-unstable MOFs include two archetypical materials, MOF- $5^{4}$ and HKUST- $1,{ }^{5}$ both widely studied as candidates for gas separation and catalysis, but whose potential for use in industrial processes could be hindered by their limited hydrothermal stability. In the case of hydrophilic HKUST-1, it is easy to see that water binds strongly to unsaturated metal sites, ${ }^{6}$ therefore leading to structural changes that ultimately lead to the framework's collapse; moreover, perfluorohexane deposition was recently demonstrated to improve the material's stability. ${ }^{7} \mathrm{MOF}-5$, however, has no coordinatively unsaturated sites and presumably little attraction to water, making it harder to understand how it can be unstable in the presence of water vapor. In this paper, we study the interactions between water and an IRMOF framework and show that although the material itself is largely hydrophobic, it is not incompatible with a favorable hydration mechanism that leads to poor hydrothermal stability. We also show how ab initio (or first principles) molecular dynamics simulations can be used to investigate the stability of metal-organic frameworks upon exposure to water, and investigate their hydration mechanism.

The materials of the IRMOF family, discovered by Yaghi and co-workers, ${ }^{4,8}$ feature oxide-centered $\mathrm{Zn}_{4} \mathrm{O}$ tetrahedral clusters as metal centers connected by dicarboxylate organic linkers and forming a 3D-connected cubic porous framework. IRMOF-1, also known as MOF-5, was the first known member of this family and is an archetypal metal-organic framework that has been widely studied and is now commercially available. Its organic linker is 1,4-benzenedicarboxylate (1,4-BDC), and it has a pore size of $\sim 9 \AA$. Other members of this rich family feature different linkers, both smaller and larger in size, some with functional groups and heteroatoms. IRMOFs in general, and MOF-5 in particular, are known to be sensitive to moisture because it is believed that the weak zinc-oxygen coordination allows for an attack by water molecules. ${ }^{9,10,11}$ Presence of water thus causes the framework to decompose, yielding lower surface area and poor adsorption properties. A number of recent publications have reported success in mitigating the issue by modifying the metal-organic framework with water-repellent functional groups, either by direct synthesis using functionalized linkers, ${ }^{12,13}$ or by postsynthetic modification of the IRMOF framework. ${ }^{14}$ Both routes allow one to tweak the hydrophobicity of the MOF, and yield materials that display almost no decline of their adsorption properties even after prolonged exposure to water. 
In addition to these functionalized materials, some groups have tried to investigate the water-MOF interactions, both experimentally and theoretically., $3,6,15,16,17,18,19$ In particular, an experimental study was reported by Sabo et al. on the conditions of hydrolysis of MOF-5 by water vapor, as well as its kinetics. ${ }^{20}$ On the theoretical side, Greathouse et al. performed a constant-pressure, forcefield-based molecular dynamics of this same material to describe its interaction with water and the influence of water content on framework deformation and stability. ${ }^{21}$ Finally, a combined experimental/theoretical study of the hydrothermal stability of various MOFs was published more recently by Low et $\mathrm{al}^{22}$ in which the authors conclude that framework stability in presence of water is mostly determined by metal-linker bond strength, which they measure using quantum mechanical calculations of MOFs cluster models. Their quantum chemistry calculations are based on the assumption that hydration takes place through linker displacement by a water molecule. In the present work, we investigate the interactions of water and the IRMOF framework in an unbiased and model-free approach, by using ab initio molecular dynamics. We previously highlighted the capabilities of this approach for the description of water adsorbed in hydrophobic and hydrophilic zeolites. $^{23,24}$ It combines the precision of quantum chemistry Density Functional Theory (DFT) calculations with a statistical sampling of phase space at non-zero temperature, allowing us to obtain more than minimum energy structures and transition states. We show here that it can thus be used to determine the hydration mechanism of a hybrid material. In addition to the ab initio MD, we have also performed forcefield-based Monte Carlo simulations, in order to ascertain the adsorption properties of IRMOFs.

\section{Computational methods}

$\mathrm{Ab}$ initio molecular dynamics of the anhydrous and hydrated MOF were performed with the CarParrinello approach ${ }^{25}$ as implemented in the CPMD software package, ${ }^{26}$ using the Kohn-Sham formulation of DFT. We employed the gradient-corrected BLYP functional. ${ }^{27,28}$ A plane-wave basis set with an energy cutoff of 90 Ry was used and only the valence electrons were explicitly considered, with semilocal norm-conserving Troullier-Martins pseudopotentials taking into account the interactions between core and valence electrons. All molecular simulations were performed using periodic boundary conditions on a single cubic unit cell of the MOF. Hydrogen atoms were deuterated in order to improve decoupling of the dynamics of the ionic and electronic subsystems and allow for a larger time step in the integration of the equations of motion. A time step of 4 a.u. $(\sim 0.1 \mathrm{fs})$ and a fictitious electron mass of 400 a.u. were used.

For each water loading simulated, a starting configuration was extracted from the previously performed grand canonical Monte Carlo simulations (see below). A first equilibration period of at least 5 ps was performed on the system in the canonical ensemble, during which the temperature 
was controlled to $300 \mathrm{~K}$ by a Berendsen thermostat (with a time constant of 1000 u.a.). ${ }^{29}$ Simulations were then run in the microcanonical NVE ensemble for respectively 185 ps (1 water molecule per unit cell) and 410 ps $\left(4 \mathrm{H}_{2} \mathrm{O}\right)$. The evolution of kinetic energy over time was checked to ensure that the NVE dynamics still corresponded to a temperature around $300 \mathrm{~K}$.

In addition to $\mathrm{ab}$ initio MD simulations, we also performed forcefield-based Monte Carlo simulations in the grand canonical ensemble. The water molecules were described by the rigid, nonpolarizable TIP4P model. The IRMOF-0h structure was described using a forcefield derived from the one Snurr et al. developed for IRMOF- $1,{ }^{30}$ where the atomic partial charges were slightly adapted to describe the acetylenedicarboxylate linker (instead of benzenedicarboxylate). These forcefield parameters are presented in the supplementary information. Lorentz-Berthelot rules were used to calculate MOF-water Lennard-Jones parameters. Series of GCMC simulations were performed at various values of water chemical potential, which was then related to water pressure by the same $\mu(P)$ relation as in ref. 24 . All Monte Carlo runs were performed at $300 \mathrm{~K}$ on a $2 \times 2 \times 2$ supercell to minimize the influence of periodic boundary conditions. Long-range electrostatic interactions were taken into account using the Ewald summation technique. In order to improve the efficiency of the calculations, electrostatic and repulsion-dispersion interaction energies between the rigid MOF framework and adsorbed water molecules were precomputed on a grid (with a grid mesh of $0.1 \AA$ ) and stored for use during the simulation.

\section{Creation and characterization of the system}

In order to characterize the hydrolysis mechanism of IRMOFs by ab initio molecular dynamics, we needed to find a member of the IRMOF family with a relatively small unit cell size so that current HPC resources would allow us to gather good statistics. IRMOF-1, also known as MOF-5, is the most studied member of the IRMOF family: its behavior in presence of water has been investigated, and functionalized MOF-5 structures have been devised that are relatively water-stable. However, with a cubic unit cell size of $25.8 \AA$ containing 424 atoms, it is much too large for our ab initio molecular dynamics study. The only known IRMOF structure with a linker shorter than IRMOF-1 is Yaghi's IRMOF-0, ${ }^{31}$ with formula $\left(\mathrm{Zn}_{4} \mathrm{O}\right)$ (acetylenedicarboxylate) $)_{3}$. However, IRMOF-0 experimentally forms as a doubly interpenetrating structure that exhibits no microporosity. We therefore created a hypothetical, non-interpenetrating analogue of IRMOF-0. We took as a starting point the experimental structure for one of the two interpenetrated networks, and ran a variable-cell DFT to minimize its energy (using the same functional, pseudopotentials and basis sets as described earlier for ab initio MD). The resulting structure is called IRMOF-0h. ${ }^{32}$ It has a cubic spacegroup $(P \overline{4} 3 m)$ and a unit cell parameter of $a=11.12 \AA$, close to the experimental value from the 
interpenetrated IRMOF-0 ( $a / 2=10.92 \AA)$. It is presented in Fig. 1, and a CIF file of the structure is provided as part of the supplementary information.

Figure 1. Representation of the cubic structure of IRMOF-0h. Carbon atoms are in black, oxygen in red, $\mathrm{Zn}$-centered tetrahedra in blue.

Grand Canonical Monte Carlo simulations of water adsorption in IRMOF-0h were then performed to characterize the affinity of water for this MOF. The adsorption-desorption isotherm cycle calculated by GCMC is shown in Fig. 2, with a change in horizontal scale at $P_{\text {sat }}=5.2 \mathrm{kPa}$ so that both the gas and liquid phase are clearly visible. This isotherm indicates that the ideal "classical" defect-free IRMOF-0h shows up as a hydrophobic material, with adsorption following a type $\mathrm{V}$ isotherm (zero slope around $P=0$ ) and a saturation uptake in excess of 30 molecules per unit cell. The hydrophobic nature of the solid is explained by the weak interactions between water and the carbon atoms of the framework, which are weaker than water-water interactions, as is the case of the archetypical water-graphene interaction. The step characteristic of the condensation transition happens at $20 \mathrm{MPa}$ upon adsorption and that of drying at $2 \mathrm{kPa}$ upon desorption, thus bracketing the water saturation pressure $P_{\text {sat }}$ with a large hysteresis loop characteristic of first-order condensationdrying transitions. ${ }^{33}$

Figure 2. Adsorption-desorption isotherms of water in IRMOF-0h calculated from Grand Canonical Monte Carlo simulations at $300 \mathrm{~K}$.

Obviously, chemical events such as bond breaking cannot take place in these simulations based on classical forcefields. The isotherm shown in Fig. 2 thus describes what would happen in IRMOF-Oh in absence of hydrolysis. We are then faced with an apparent contradiction. IRMOF-0h appears as a hydrophobic solid, while in real life the IRMOF materials are water unstable ${ }^{8}$. An examination of the water adsorption studies in several MOFs by Kaskel and coworkers ${ }^{9}$ enables to understand that the hydrophilic/hydrophobic character of the inner surface of the MOF material should not be confused with the instability/resistance with respect to hydration. Indeed, these authors observe a Type I water adsorption isotherm in the case of HKUST-1, on account of the hydrophilicity of the free copper sites, accompanied by chemisorption and hydrolysis. In ZIF-8, a type V isotherm is observed, with a "remarkable chemical stability towards water". In the intermediate DUT-4 case, the adsorption branch of the isotherm is of type $\mathrm{V}$, but the desorption branch shows that this 
aluminum based material, in which there are no free coordination sites available, experiences decomposition at high water loading. It turns out then that a hydrophobic MOF can be water unstable, a feature that was overlooked in some previous studies. ${ }^{6,7,8}$ The following ab initio simulations provide some insight into this phenomenon.

\section{Ab initio molecular dynamics at low water loading}

A first ab initio simulation was run with a single water molecule adsorbed in a unit cell of IRMOF$0 \mathrm{~h}$, for a total of $185 \mathrm{ps}$ of NVE molecular dynamics. For most of the trajectory's length, the water molecule diffuses rapidly in the nanopores of the material. However, there are also periods of time during which it gets closer to one zinc ion (down to $2 \AA$, see fig. 3). The histogram of $\mathrm{Zn}-\mathrm{O}$ distances (fig. 4) shows a clear peak at about $2.2 \AA$. This state corresponds to the water molecule being bound to $\mathrm{Zn}^{2+}$ ion, i.e. entering its coordination complex.

Figure 3. Distance between the oxygen of the water molecule and the closest $\mathrm{Zn}^{2+}$ ion in a simulation of one $\mathrm{H}_{2} \mathrm{O}$ in IRMOF-0h at $300 \mathrm{~K}$. The threshold of $2.8 \AA$ chosen to characterize the bound state of the water molecule is indicated as a red dashed line. Each occurrence of a bound state during the dynamics is indicated by a thick horizontal line, labelled with the index of the $\mathrm{Zn}^{2+}$ ion involved.

Figure 4. Histogram of water-Zn distance for one water molecule adsorbed in IRMOF-0h at $300 \mathrm{~K}$ (upper panel) and potential of mean force (lower panel).

To further characterize this state during the simulation, we decided on a simple geometric threshold at the minimum in the distribution of distances: we consider that $d(\mathrm{O}-\mathrm{Zn}) \leq 2.8 \AA$ indicates a bound state, while larger distances correspond to a free water molecule. With this criterion, we see that over the 160 ps of the dynamics, three events of water attack on the metal centre occurred. Each of the bound states produced (see Table 1) are relatively short-lived, lasting no more than 3.0 ps (see Table 2). Hence, water attack on the zinc cation is a relatively frequent event, is reversible, and the free $\rightleftarrows$ bound equilibrium strongly favors the free state at this low water loading. The existence of this bound state is consistent with the hydrophobic nature of the host matrix and its type $\mathrm{V}$ isotherm. Indeed, hydrophobicity does not mean there are no interactions (or purely repulsive interactions), but only that host-guest interactions are weaker than guest-guest interactions. ${ }^{33}$ Hence it is possible for a bound state to exist, but it must be thermodynamically unfavourable compared to the freely diffusing molecule for the host to be considered hydrophobic. 
In order to quantify this bound state further, we computed the potential of mean force as a function of $\mathrm{Zn}-\mathrm{O}_{\text {water }}$ distance from our dynamics. While this certainly bears some uncertainty due to the relatively small statistical sampling compared to the frequency of the reaction, it does give an estimate of the free energy difference between the two states, and the barrier height. The calculated potential of mean force is plotted on Fig. 4. It shows a free energy barrier for the attack of $\Delta \mathrm{F}^{\neq}=$ $3.2 k T(\sim 8 \mathrm{~kJ} / \mathrm{mol})$ and a free energy difference between metastable states of $1.3 k T(\sim 3.2 \mathrm{~kJ} / \mathrm{mol})$ in favor of the water-free state.

Furthermore, we characterized this state of one water molecule bound to the $\mathrm{Zn}^{2+}$ ion. The cation has a tetrahedral environment in the nonhydrated material (state $\mathbf{A}$ in the overall sketch on Fig. 5), with 4 oxygen atoms coordinated to it: 3 from the carboxylate linkers, and 1 being the central $\mathrm{O}$ atom of the $\mathrm{Zn}_{4} \mathrm{O}$ cluster. When the water molecule binds to the $\mathrm{Zn}^{2+}$, none of these atoms exit the coordination shell, and the $\mathrm{Zn}^{2+}$ thus has a trigonal bipyramidal environment (state $\mathbf{B}$ in Fig. 5). This is rather typical of $\mathrm{Zn}(\mathrm{II})$, which is commonly encountered in high-coordination structures, including pentacoordinate states. ${ }^{34,35}$ This structure is moreover similar in geometry to the "hydrated" state hypothesized by Low et al. ${ }^{22}$ as the initial state of their ligand displacement mechanism. We further confirmed that this state is indeed a stable state, and obtained its geometric characteristics (given in Table 1) by an energy minimization with a variable cell (using the same DFT parameters as for the MD run).

Figure 5. Different states of a $\mathrm{Zn}^{2+}$ coordination sphere of IRMOF-0h with zero or one bound water molecules. See text for details on each state.

\begin{tabular}{|c|c|c|c|}
\hline & $\mathbf{A}$ & $\mathbf{B}$ & $\mathbf{C}$ \\
\hline geometry & tetrahedral & $\begin{array}{c}\text { trigonal } \\
\text { bipyramid }\end{array}$ & tetrahedral \\
\hline$d\left(\mathrm{Zn}-\mathrm{O}_{\text {central }}\right)$ & $2.00 \AA$ & $1.98 \AA$ & $2.04 \AA$ \\
\hline$d\left(\mathrm{Zn}-\mathrm{O}_{\text {linker }}\right)$ & $2.00 \AA$ & $2.04 \AA$ & $1.97 \AA$ \\
\hline$d\left(\mathrm{Zn}-\mathrm{O}_{\text {water }}\right)$ & - & $2.06 \AA$ & $2.17 \AA$ \\
\hline
\end{tabular}

Table 1. Geometrical characteristics of $\mathrm{Zn}^{2+}$ environment each state described in Fig. 5.

\section{Effect of higher water loading}

In order to investigate the effect of hydration level on the stability of IRMOFs, we ran ab initio MD simulations with a loading of 4 water molecules per unit cell of IRMOF-0h. The attacks of water 
molecules on the $\mathrm{Zn}_{4} \mathrm{O}$ metal cluster observed for 1 and 4 water molecules, and the lifetimes of the bound states produced, are presented in Table 2. It is clear that the presence of additional water molecules in the IRMOF-0h unit cell stabilizes the bound state and displaces the $\mathbf{A} \rightleftarrows \mathbf{B}$ equilibrium towards water-bound state B, precursor of hydrolysis. For the 4 water molecules system, which ran for a total of $410 \mathrm{ps}$, a single water attack event was observed at $34.5 \mathrm{ps}$, after which the bound state B was never unformed. Because of this single event, we could not estimate the free energy difference between the $\mathbf{A}$ and $\mathbf{B}$ states at a water loading of $4 \mathrm{H}_{2} \mathrm{O} /$ u.c., but only conclude that $\mathbf{B}$ is much more stable than $\mathbf{A}$ in these conditions.

To analyse the reasons for this stabilization, we characterized the hydrogen bonds network of the adsorbed water. We used a simple geometric criterion: ${ }^{36} d_{\mathrm{OO}} \leq 3.5 \AA, \theta(\mathrm{OOH}) \leq 40^{\circ}$. During all the time spent with a water molecule bound to $\mathrm{Zn}^{2+}$ (state $\mathbf{B}$ ), the other three water molecules in the system tend to stay in the vicinity of the bound molecule, forming a weak hydrogen bonds network. Over the course of the entire simulation (276 ps), this hydrogen bonds network reorganizes several times, leading to various geometries featured in Fig. S2. However, we observed at least one water molecule hydrogen-bonded to the bound one at all times. The existence of this network of water molecules accounts for the stabilization of the bound $\mathrm{Zn}-\mathrm{H}_{2} \mathrm{O}$ state with increasing water loading.

1 water molecule (total: $185 \mathrm{ps)}$

\begin{tabular}{|c|c|c|c|c|}
\hline $\mathrm{H}_{2} \mathrm{O}$ index & Zn index & Attack time & $\begin{array}{l}\text { Departure } \\
\text { time }\end{array}$ & $\begin{array}{l}\text { Lifetime of } \\
\text { bound state }\end{array}$ \\
\hline $\mathrm{H}_{2} \mathrm{O}(1)$ & $\mathrm{Zn}(2)$ & $47.5 \mathrm{ps}$ & $50.5 \mathrm{ps}$ & $3.0 \mathrm{ps}$ \\
\hline $\mathrm{H}_{2} \mathrm{O}(1)$ & $\mathrm{Zn}(4)$ & $105.0 \mathrm{ps}$ & $105.3 \mathrm{ps}$ & $0.3 \mathrm{ps}$ \\
\hline $\mathrm{H}_{2} \mathrm{O}(1)$ & $\mathrm{Zn}(1)$ & $117.0 \mathrm{ps}$ & $118.4 \mathrm{ps}$ & $1.4 \mathrm{ps}$ \\
\hline \multicolumn{5}{|c|}{4 water molecules (total: $410 \mathrm{ps)}$} \\
\hline \multirow[t]{12}{*}{$\mathrm{H}_{2} \mathrm{O}$ index } & $\mathrm{Zn}$ index & Attack time & $\begin{array}{l}\text { Departure } \\
\text { time }\end{array}$ & $\begin{array}{l}\text { Lifetime of } \\
\text { bound state }\end{array}$ \\
\hline & & $34.5 \mathrm{ps}$ & - & $\geq 375 \mathrm{ps}$ \\
\hline & & $\begin{array}{l}\text { State B (trigonal } \\
\text { bipyramid) }\end{array}$ & & \\
\hline & & $34.5 p s$ & $97 p s$ & $62.5 \mathrm{ps}$ \\
\hline & & $206 \mathrm{ps}$ & $222 p s$ & $16.0 \mathrm{ps}$ \\
\hline & & $264 p s$ & $282 p s$ & $18.0 \mathrm{ps}$ \\
\hline & & $311 p s$ & $338 p s$ & $27.0 \mathrm{ps}$ \\
\hline & & $\begin{array}{c}\text { State C } \\
\text { (tetrahedral) }\end{array}$ & & \\
\hline & & $97 p s$ & $206 p s$ & $109 \mathrm{ps}$ \\
\hline & & $222 p s$ & $264 p s$ & $42 p s$ \\
\hline & & $282 p s$ & $311 p s$ & $29 p s$ \\
\hline & & $338 p s$ & - & $\geq 72 p s$ \\
\hline
\end{tabular}


Table 2. Analysis of the attacks of water molecules on $\mathrm{Zn}^{2+}$ ions, and the lifetime of the zinc-water bound states.

Besides the bipyramidal water-bound state observed upon $\mathrm{H}_{2} \mathrm{O}$ attack of the metal center, the ab initio MD for 4 adsorbed water molecules revealed a second hydrated state of the IRMOF-0h. In this state, labeled $\mathbf{C}$ and represented in Fig. 6, one of the carboxylate linker is no longer bound to the $\mathrm{Zn}^{2+}$ cation, which thus regains its tetrahedral coordination (water molecule, $\mathrm{Zn} 4 \mathrm{O}$ central oxygen and 2 carboxylate oxygens). Because this state has a carboxylate linker no longer coordinated to two metal cations, i.e. with a "dangling oxygen", it clearly constitutes a second step in the hydration mechanism (Fig. 5), i.e. the displacement of the linker. The $\mathbf{C}$ state is short-lived and the second reaction $(\mathbf{B} \rightleftarrows \mathbf{C}$ ) is again reversible (see Table 2 ), and in fact in rapid equilibrium between what can be seen as two labile states. Moreover, we observe from the evolution of the various $\mathrm{Zn}-\mathrm{O}_{\text {carboxylate }}$ distances pictured over time on Fig. S3 that the linker displaced by the water molecule is not the same in every attack. This hydrated state can thus be described as a dynamic equilibrium $\mathbf{B} \rightleftarrows \mathbf{C}_{1} \rightleftarrows B \rightleftarrows \mathbf{C}_{2} \rightleftarrows \mathbf{B} \rightleftarrows \mathbf{C}_{3}$. We expect that this situation would be somewhat modified in a real experimental system of macroscopic size: in constant-pressure conditions, the deformation of the unit cell gives the linker full latitude to move, and if a system larger than a single unit cell were considered, more than one metal cluster could be attacked by water. As a consequence, the linker once displaced would later move away from the zinc structure, eventually leading to the collapse of the structure.

Figure 6. Snapshot of a water molecule bound to the $\mathrm{Zn}^{2+}$ ion of IRMOF-0h, with tetrahedral coordination (hydrated state $\mathbf{C}$ ). The other 3 water molecules which form a hydrogen-bonded network with the first one (see text) are not shown.

We further characterized the equilibrium between two states by monitoring the evolution of the $\mathrm{O}_{\mathrm{c}} \mathrm{ZnO}_{\text {water }}$ angle along the simulation: the bipyramidal state $\mathbf{B}$ corresponds to values close to $180^{\circ}$ (the water and central oxygen atoms being on apical positions), while in the almost tetrahedral state C this angle is closer to $109.4^{\circ}$. The system can clearly be seen to switch between these two welldefined states (Fig. S3). The potential of mean force along this reaction coordinate is featured in Figure 7, which confirms the small free energy barrier between the two states. It also indicates that under these conditions, structure $\mathbf{C}$ with the displaced ligand is slightly favored over hydrated state B. 
Figure 7. Potential of mean force as a function of $\mathrm{O}_{c} \mathrm{ZnO}_{\text {water }}$ angle in the MD simulation of $4 \mathrm{H}_{2} \mathrm{O}$ molecules in IRMOF-0h at $300 \mathrm{~K}$, showing the equilibrium between states $\mathbf{B}$ and $\mathbf{C}$.

\section{Overall mechanism and comparison with previous studies}

The overall mechanism that we derived from our ab initio MD simulations is presented above in Fig. 5. It is important to note that this mechanism is not an hypothesis of our work, but was derived entirely from direct first-principles simulations at various water loading. Hydration proceeds in two successive steps: (i) water binding to the metallic cluster; (ii) linker displacement, where one water molecule actually replaces one of the linker arms. These two steps take place very rapidly, since they can be observed on the timescale of ab initio MD, provided that there is enough water present in the porous framework (here 4 molecules per unit cell corresponds to 12 weight\%). One water molecule binds to the metallic cluster and the extra water molecules stabilize both the hydrated and the linker-displaced states so that the energy barrier for the hydration reactions become of the order or less than $k T$. Thus, we showed that the hydration and linker displacement mechanisms cannot be well-described by cluster calculations with a single water molecules: a full unit cell has to be used, and the influence of the material's water loading need to be taken into account. The studies of ref 22 for instance were performed with a single water molecule and gave a very high value of the energy barrier for linker displacement $\left(\Delta E^{\neq} \sim 48.6 \mathrm{~kJ} / \mathrm{mol}\right)$.

Finally, the comparison of the present work with the results of previous theoretical work by Greathouse et $\mathrm{al}^{21}$, who performed constant pressure forcefield-based classical MD of hydrated IRMOF-1, reveals stark differences in the structures obtained for the bound $\mathrm{Zn}^{2+}$-water states. From the results of their forcefield-based simulations, Greathouse et al concluded that water displaced rather indifferently two types of oxygen atoms initially coordinated to the $\mathrm{Zn}^{2+}$ ions: the central atom of the $\mathrm{Zn}_{4} \mathrm{O}$ tetrahedron, which we call $\mathrm{O}_{\text {central, }}$ and those of the linkers' carboxylate groups, $\mathrm{O}_{\text {linker }}$. This contrasts with our results presented above, where we characterized two possible hydrated states at low to medium water loading, none of which displays a rupture (or even an marked elongation) of the $\mathrm{Zn}-\mathrm{O}_{\text {central }}$ bond. In fact, this bond appears to be the stronger of the two coordinative bonds, and the $\mathrm{Zn}^{2+}$ cation will prefer keeping a coordination number of five, with a trigonal bipyramid geometry, rather than break the $\mathrm{Zn}-\mathrm{O}_{\text {central }}$ bond. We believe this difference with Greathouse's findings is due to a bias of the classical forcefield, which has a tendency to describe the $\mathrm{Zn}^{2+}$ as tetrahedrally coordinated. 


\section{Conclusions}

The inner surface of porous IRMOFs, modelled in this work by the smaller IRMOF-0h, mainly exhibit non polar functional groups together with $\mathrm{Zn}$ ions that are coordinated to oxygen atoms. This surface has an overall hydrophobic character, and this explains the type V water adsorption isotherm observed in a classical Monte Carlo simulation. The $\mathrm{Zn}_{4} \mathrm{O}$ clusters act as "weak hydrophilic defects", as defined in ref. 37 and 38. When only one water molecule is present in the pore, the bound state is frequently visited but it is less stable than the water free state, and no chemisorption of any kind is taking place. At a higher water loading, a water cluster can be formed at the $\mathrm{Zn}$ cluster site and this was shown to stabilize the water bound state, which very rapidly transforms into a linker-displaced state, where water has fully displaced one arm of a linker and which corresponds to the loss of the material's fully-ordered structure. Thus an overall hydrophobic MOF material can also become water unstable, a feature that was not fully understood until now.

In future works, we aim at extending our ab initio molecular dynamics simulations to the isothermal-isobaric $(N, P, T)$ ensemble with a varying unit cell, so that the complete hydration event could be simulated. This kind of study will be extended to other families of metal-organic frameworks, in order to evaluate the influence of metal-linker coordination chemistry on the stability of these materials. We will also investigate the stabilization of functionalized IRMOF variants such as $\mathrm{CH}_{3}$-MOF-5, for which the steric effect of the methyl group is not enough to justify the increased hydrothermal stability of the material.

\section{Acknowledgments}

The authors thank Rodolphe Vuilleumier and Anne Boutin for their technical expertise and many fruitful discussions. This work was supported by the Agence Nationale de la Recherche under contract ANR-2010-BLAN-0822-02, and performed using HPC resources from GENCI-IDRIS (project 096114).

\section{Notes and References}

${ }^{1}$ J.-R. Li, R. J. Kuppler, H.-C. Zhou, Chem. Soc. Rev. 2009, 38, 1477-1504.

${ }^{2}$ A. U. Czaja, N. Trukhan, U. Müller, Chem. Soc. Rev. 2009, 38, 1284-1293.

${ }^{3}$ P. Küsgens, M. Rose, S. Senkovska, H. Fröde, A. Henschel, S. Siegle, S. Kaskel, Micro. Meso.

Mater. 2009, 120, 325-330. 
${ }^{4}$ H. Li, M. Eddaoudi, M. O’Keeffe, O. M. Yaghi, Nature 1999, 402, 276-279.

${ }^{5}$ S.-Y. Chui, M.-F. Lo, J. P. H. Charmant, A. G. Orpen, I. D. Williams, Science 1999, 283, 1148 1150 .

${ }^{6}$ A. O. Yazaydin, A. I. Benin, S. A. Faheem, P. Jakubczak, J. J. Low, R. R. Willis, R. Q. Snurr, Chem. Mater. 2009, 21, 1425-1430.

${ }^{7}$ J. B. Decoste, G. W. Peterson, M. W. Smith, C. A. Stone, C. R. Willis, J. Am. Chem. Soc. 2012, 134, 1486-1489.

${ }^{8}$ M. Eddaoudi, J. Kim, N. Rosi, D. Vodak, J. Wachter, M. O’Keeffe, O. M. Yaghi, Science 2002, 295, 469-472.

${ }^{9}$ L. M. Huang, H. T. Wang, J. X. Chen, Z. B. Wang, J. Y. Sun, D. Y. Zhao, Y. S. Yan, Micro. Meso. Mater. 2003, 58, 105.

${ }^{10}$ S. S. Kaye, A. Dailly, O. M. Yaghi, J. R. Long, J. Am. Chem. Soc. 2007, 129, 14176.

${ }^{11}$ S. Hausdorf, J. Wagler, R. Mossig, F. Mertens, J. Phys. Chem. A, 2008, 112, 7567.

12 J. Yang, A. Grzech, F. M. Mulderb, T. J. Dingemans, Chem. Commun. 2011, 47, 5244.

${ }^{13}$ T. Wu, L. Shen, M. Luebbers, C. Hu, Q. Chen, Z. Ni, R. I. Masel, Chem. Commun. 2010, 46, 6120 .

${ }^{14}$ J. G. Nguyen, S. M. Cohen, J. Am. Chem. Soc. 2010, 132, 4560.

${ }^{15}$ K. Schröck, F. Schröder, M. Heyden, R. A. Fischer, M. Havenith, Phys. Chem. Chem. Phys. 2008, 10, 4732-4739.

${ }^{16}$ S. Paranthaman, F.-X. Coudert, A. H. Fuchs, Phys. Chem. Chem. Phys. 2010, 12, 8123-8129.

${ }^{17}$ S. Bourrelly, A. Vimont, G. Maurin, S. Devautour-Vinot, T. Devic, P. Horcajada, C. Serre, G. Clet, M. Daturi, Y. Filinchuk, G. Férey, P. L. Llewellyn, J. Am. Chem. Soc. 2010, 132, 9488.

${ }^{18}$ F. Gul-E-Noor, B. Jee, A. Pöppl, M. Hartmann, D. Himsl, M. Bertmer, Phys. Chem. Chem. Phys. 2011, 13, 7783-7788.

${ }^{19}$ Y. Cheng, A. Kondo, H. Noguchi, H. Kajiro, K. Urita, T. Ohba, K. Kaneko, H. Kanoh, Langmuir, 2009, 25, 4510-4513.

${ }^{20}$ M. Sabo, A. Henschel, H. Fröde, E. Klemm, S. Kaskel, J. Mater. Chem. 2007, 17, 3827.

${ }^{21}$ J. A. Greathouse, M. D. Allendorf, J. Am. Chem. Soc. 2006, 128, 10678.

22 J. J. Low, A. I. Benin, P. Jakubczak, J. F. Abrahamian, S. A. Faheem, R. R. Willis, J. Am. Chem. Soc. 2009, 131, 15834-15842.

${ }^{23}$ F.-X. Coudert, R. Vuilleumier, A. Boutin, Chem. Phys. Chem. 2006, 7, 2464-2467.

${ }^{24}$ F.-X. Coudert, F. Cailliez, R. Vuilleumier, A. H. Fuchs, A. Boutin, Faraday Discuss. 2009, 141, 377.

${ }^{25}$ R. Car, M. Parrinello, Phys. Rev. Lett. 1985, 55, 2471.

${ }^{26}$ CPMD, http://www.cpmd.org/, Copyright IBM Corp 1990-2008, Copyright MPI für Festkörperforschung Stuttgart 1997-2001.

${ }^{27}$ A. D. Becke, Phys. Rev. A 1988, 38, 3098.

${ }^{28}$ C. Lee, W. Yang, R. G. Parr, Phys. Rev. B 1988, 37, 785.

${ }^{29} \mathrm{We}$ initially tried to perform our ab initio dynamics in the NPT ensemble, which fails because of a too high computational cost. The very large planewave cutoff required to correctly converge the stress on the unit cell was estimated at 250 to $300 \mathrm{Ry}$, and the fluctuations in unit cell volume were too large for one unit cell, making the system unstable. The use of a supercell (at least $2 \times 2 \times 2$, possible more) would make the system too large for ab initio MD. So, we focused instead on dynamics in the NVT ensemble. 
${ }^{30}$ K. S. Walton, A. R. Millward, D. Dubbeldam, H. Frost, J. J. Low, O. M. Yaghi, R. Q. Snurr, J. Am. Chem. Soc. 2008, 130, 406-407.

${ }^{31}$ D. J. Tranchemontagne, J. R. Hunt, O. M. Yaghi, Tetrahedron 2008, 64, 8553.

${ }^{32}$ D. Fairen-Jimenez, N. A. Düren T. Seaton, Langmuir 2010, 26, 14694-14699.

${ }^{33}$ F. Cailliez, M. Trzpit, M. Soulard, I. Demachy, A. Boutin, J. Patarin, A.H. Fuchs, Phys. Chem. Chem. Phys. 2008, 10, 4817-4826.

${ }^{34}$ S. Bhattacharyya, S. B. Kumar, S. K. Dutta, E. R. T. Tiekink, M. Chaudhury, Inorg. Chem. 1996, 35, 1967-1973.

${ }^{35}$ H. Nekola, D. Rehder, Inorg. Chim. Acta 2002, 337, 467-469.

${ }^{36}$ A. Luzar, D. Chandler, Nature 1996, 379, 55.

${ }^{37}$ M. Trzpit, M. Soulard, J. Patarin, N. Desbiens, F. Cailliez, A. Boutin, I. Demachy, A. H. Fuchs, Langmuir 2007, 23, 10131-10139.

${ }^{38}$ F. Cailliez, G. Stirnemann, A. Boutin, I. Demachy, A. H. Fuchs, J. Phys. Chem. C 2008, 112, 10435-10445. 


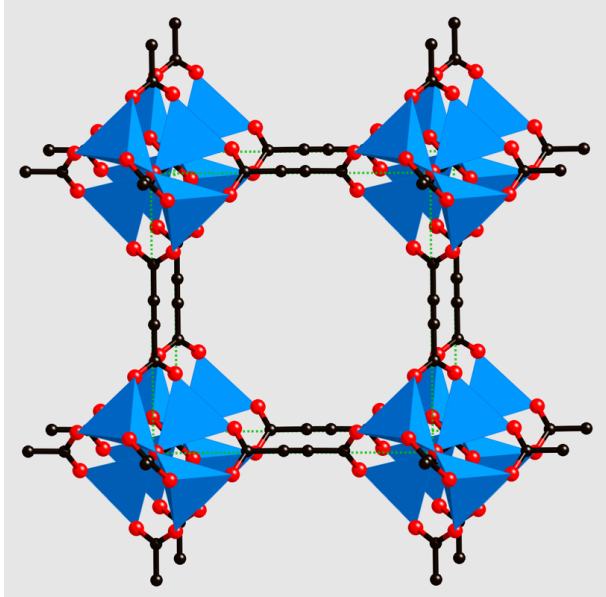

Figure 1 


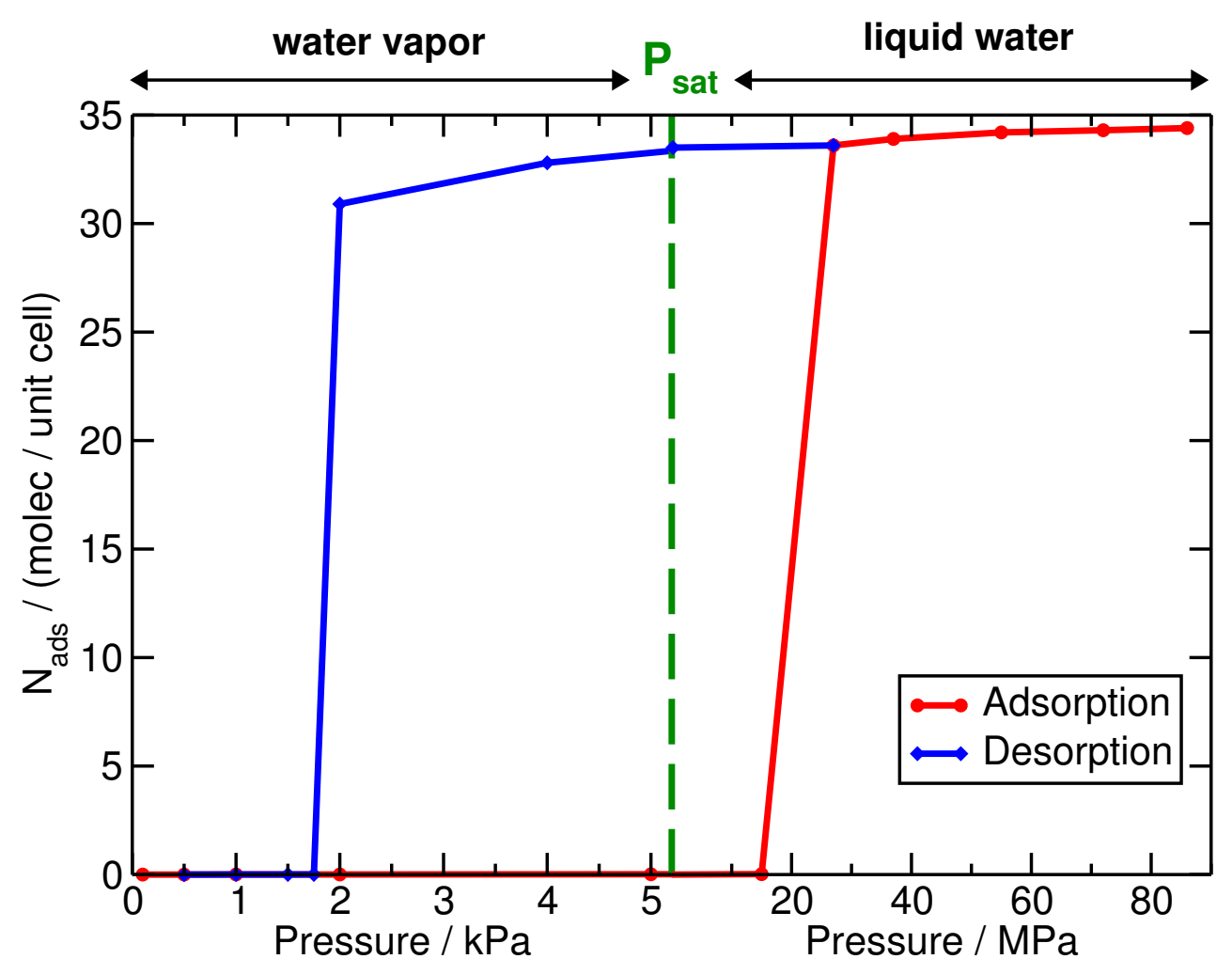

Figure 2 


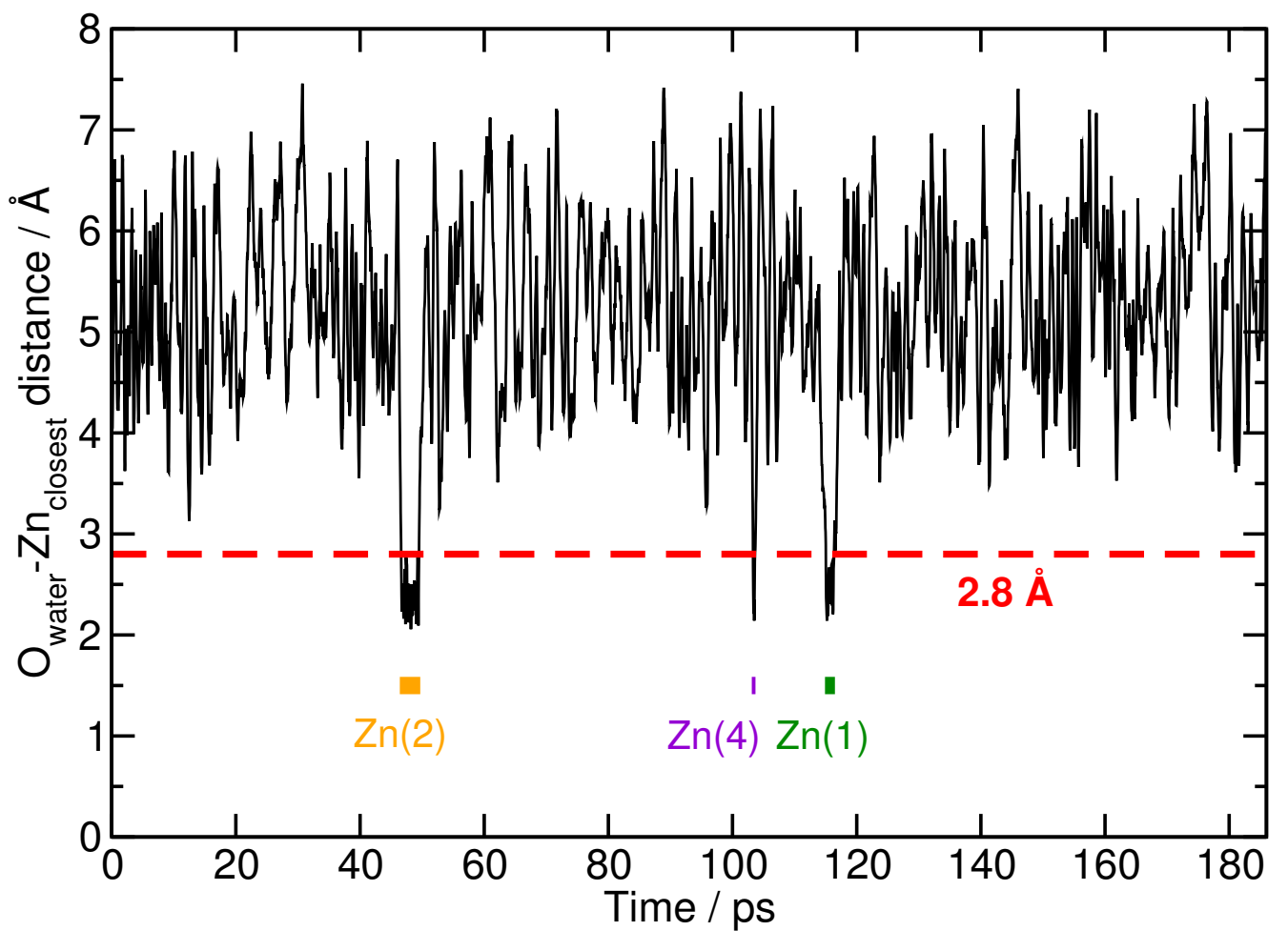

Figure 3 


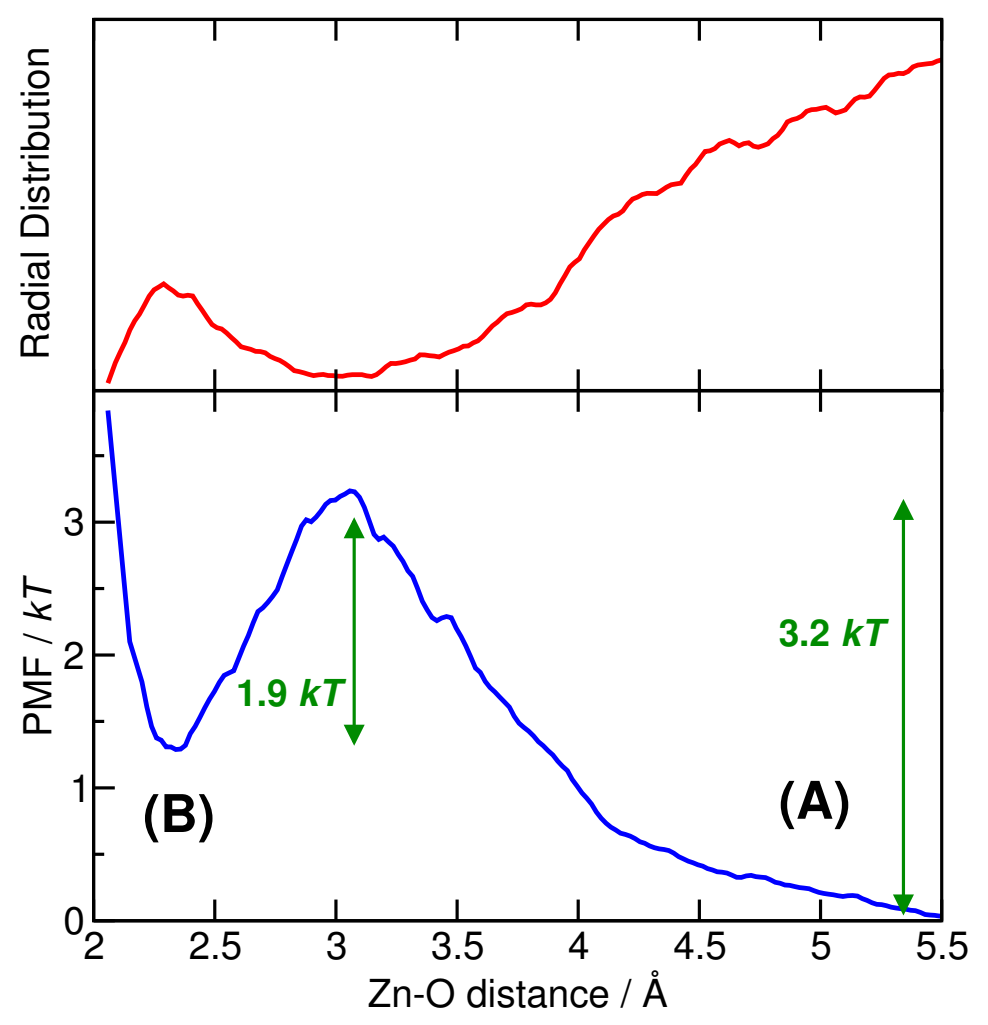

Figure 4 


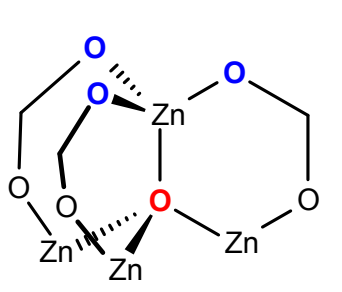

(A)

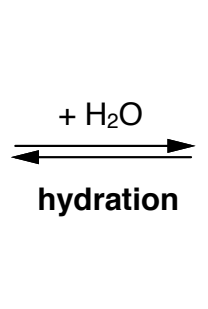

dry IRMOF-0h

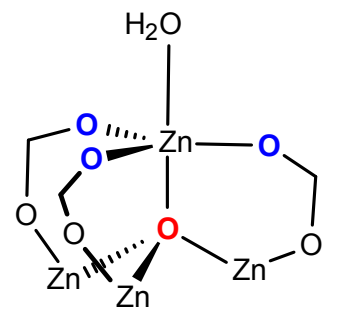

(B)

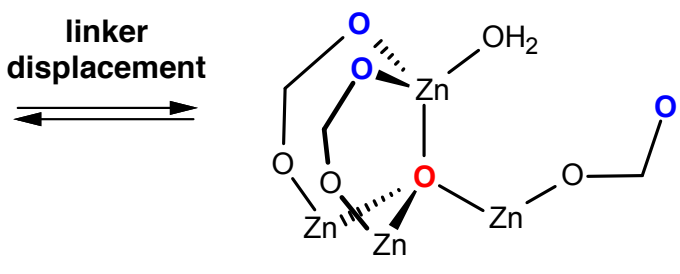

(C)

Figure 5 


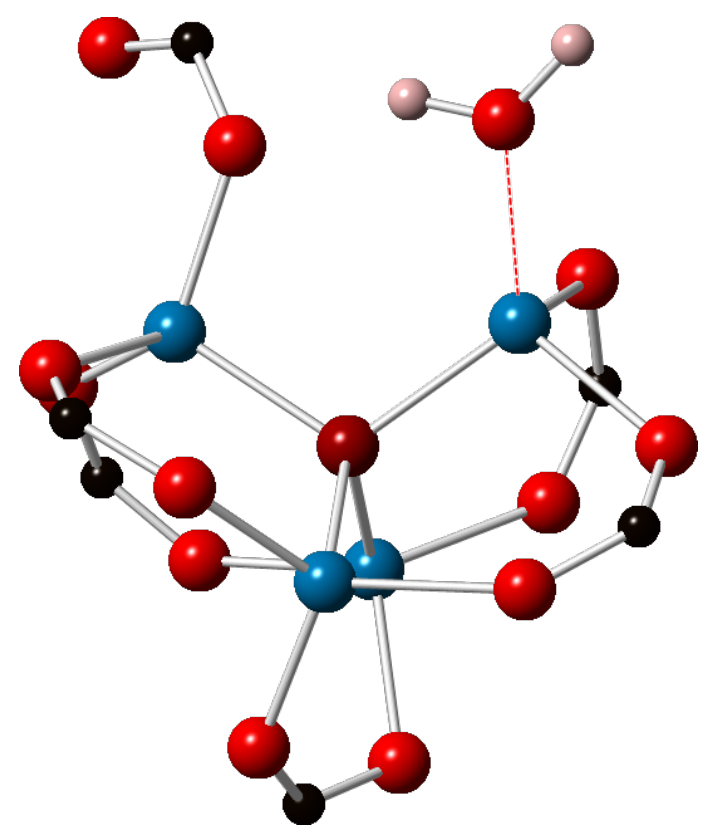

Figure 6 


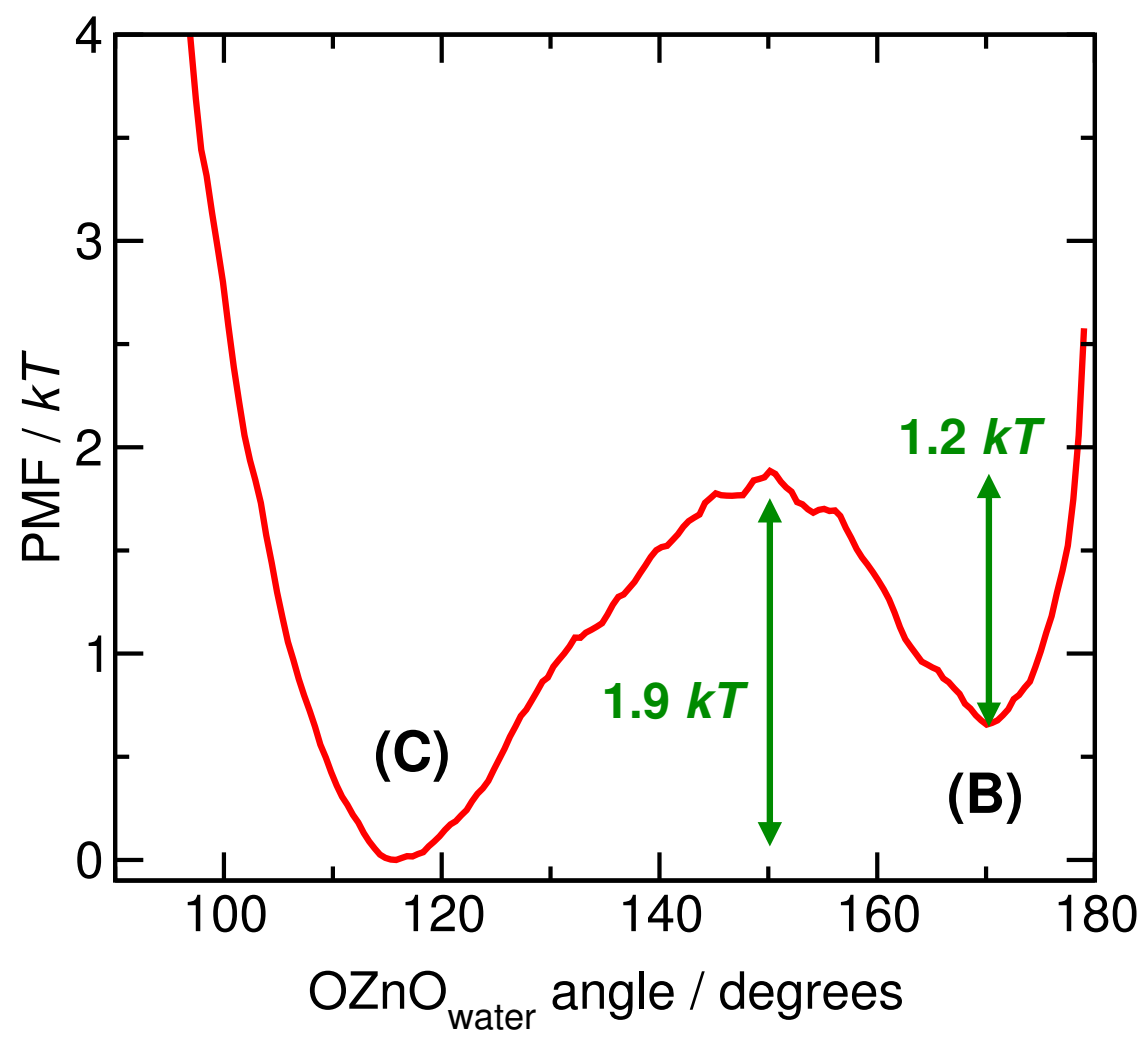

Figure 7 
Table of Contents entry

\section{IRMOF hydration mechanism}

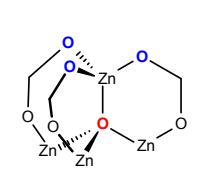

(A)

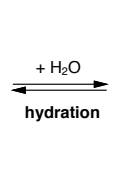

dry IRMOF-0h

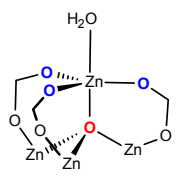

(B)

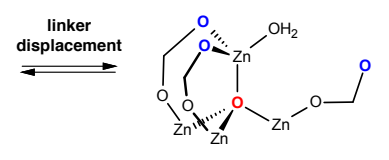

(C)

$\mathrm{Ab}$ initio molecular dynamics gives insight into the hydration mechanism of metal-organic frameworks from the IRMOF family, showing how they can be both hydrophobic and waterunstable. 